\title{
Clasificación de humedales de montaña (Vegas) en los Andes Centrales de Argentina
}

\author{
Classification of mountain wetlands (Vegas) in the Central Andes, \\ Argentina
}

\author{
Marcela Ontivero ${ }^{1,2 *}\left(\mathbb{C}\right.$, Eduardo Martínez Carretero ${ }^{1}\left[0\right.$ y Laura Perucca ${ }^{3}[$
}

1. Grupo de Geobotánica y Fitogeografía- Instituto Argentino de Investigación de Zonas Áridas, Consejo Nacional de Investigaciones Científicas y Técnicas, CP 5500, Mendoza, Argentina.

2. Centro de Fotogrametría Cartografía y Catastro. Facultad de Ingeniería. Universidad Nacional de San Juan. Laprida 1130 (O) CP 5400, San Juan, Argentina.

3. Gabinete de Neotectónica y Geomorfología, INGEO- Facultad de Ciencias Exactas, Físicas y Naturales. Universidad Nacional de San Juan. Av. Ignacio de la Roza 590, CP 5400, San Juan, Argentina.

*montivero@mendoza-conicet.gob.ar

\section{Citar este artículo}

ONTIVERO, M., E. MARTÍNEZ CARRETERO \& L. PERUCCA. 2022. Clasificación de humedales de montaña (Vegas) en los Andes Centrales de Argentina. Bol. Soc. Argent. Bot. 57: 51-63.

DOI: https://doi. org/10.31055/1851.2372.v57. n1.33884

Recibido: 12 Jul 2021

Aceptado: 15 Nov 2021

Publicado en línea: 20 Feb 2022

Publicado impreso: 31 Mar 2022

Editor: Ramiro Aguilar (D)

ISSN versión impresa 0373-580X

ISSN versión on-line 1851-2372

\section{SUMMARY}

Background and aims: The objective of this work is to classify mountain wetlands (vegas) in the Central Andes between $28^{\circ}$ y $53^{\circ} \mathrm{S}$, based on geomorphology and hydrology. In addition, it seeks to characterize and determine the area, height, and slope of the vegas in each typology.

M\&M: The determination of the different typologies was carried out based on an integrated biophysical approach at different scales of spatial analysis, where the vegas present in the study area were identified and their link with hydrological and geomorphological aspects. The dominant runoff types, and the geomorphological units were determined. Satellite images ALOS- AVNIR-2, Landsat 5 TM and field data were used.

Results: A total of 304 vegas were identified that are determined by the geomorphological unit (river plains, alluvial fan, hillside, undifferentiated foothills, depression without surface discharge with or without water and fault) and the dominant type of runoff (linear surface, mantiform subsurface and subsurface mantiform to superficial linear). Four typologies of vegas are recognized: Riverbank, Hillside, Depression and Fault. The Riverbank and Hillside typologies were dominant in number and area. Eight and slope did not result different between typologies.

Conclusions: The vegas present in the Central Andes respond to 4 typologies: Riverbank, Fault, Hillside and Depression. There is a close relationship between vegas, geomorphology and the dominant runoff, allowing their classification and to analyze different management measures. The obtained results are a contribution to the study of ecosystems of high ecological and socio-economic value in the region.

\section{KeY Words}

Argentina, Central Andes, classification, geomorphology, typology, vegas, wetlands

\section{RESUMEN}

Introducción y objetivos: El objetivo de este trabajo fue clasificar las vegas en los Andes Centrales entre los $28^{\circ}$ y $53^{\circ} \mathrm{S}$, con base en la geomorfología y la hidrología. Además, se buscó caracterizar las vegas del área de estudio en base a la superficie, altura y pendiente según tipología.

M\&M: La determinación de las distintas tipologías se realizó en base a un enfoque biofísico integrado a distintas escalas espaciales, donde se identificó las vegas y se determinó su vinculación con la hidrología y geomorfología. Se determinaron los tipos de escurrimientos dominantes y las unidades geomorfológicas. Se utilizaron imágenes satelitales ALOS- AVNIR-2, Landsat 5 TM y datos de campo.

Resultados: Se identificaron 304 vegas que están determinadas por la unidad geomorfológica (planicies fluviales, abanico aluvial, ladera, piedemonte indiferenciado, depresión sin descarga superficial con o sin agua y falla) y el tipo de escurrimiento dominante (superficial lineal, subsuperficial mantiforme y subsuperficial mantiforme a superficial lineal). Se reconocen cuatro tipologías de vegas: Ribera, Ladera, Depresión y Falla. La tipología de Ribera y de Ladera fueron las dominantes en cuanto a número y superficie. La altura y la pendiente no resultaron diferentes entre tipologías.

Conclusiones: Las vegas en los Andes Centrales responden a 4 tipologías: Ribera, Falla, Ladera y Depresión. Existe una estrecha relación entre las vegas, la geomorfología y el escurrimiento dominante, lo que permite clasificarlas y analizar distintas medidas de manejo. Los resultados obtenidos son un aporte al estudio de ecosistemas de alto valor ecológico y socio-económico en la región.

\section{Palabras Claves}

Andes Centrales, Argentina, clasificación, geomorfología, humedales, tipología, vegas. 


\section{INTRODUCCIÓN}

El término humedal comprende un conjunto de ambientes muy diversos que integran áreas inundadas o saturadas por aguas superficiales o subterráneas, de manera permanente o temporal, con una frecuencia y duración suficiente para soportar, bajo condiciones normales, vegetación predominantemente adaptada a condiciones de suelos saturados (Mitsch \& Gosselink, 2007; Keddy, 2010). De acuerdo con Brinson (1993), la localización y distribución de los humedales está dada por su emplazamiento geomorfológico y responde, principalmente a factores topográficos, hidrológicos y climáticos particulares. Las condiciones abióticas son importantes en el mantenimiento de la estructura $\mathrm{y}$ el funcionamiento de este tipo de ecosistemas, ya que determinan condiciones en la riqueza, composición de especies y productividad primaria (Mitsch \& Gosselink, 2007).

El inventario de humedales, así como su clasificación en distintas tipologías, es un paso previo para la elaboración de programas de conservación y gestión (Finlayson et al., 1999). Los factores responsables de controlar el rango de condiciones en el ambiente de los humedales son mucho más variables que lo que sucede en los ecosistemas terrestres. De esta forma, los humedales tienen una compleja diversidad de elementos, ambientes y funciones, que hace necesario otorgar un orden a través de una clasificación en función de sus principales características, lo que permite profundizar en las investigaciones sobre su estructura y funcionamiento (Brinson, 2004a, Malvárez, 2004;).

En la clasificación e inventario de humedales existen amplios criterios sobre cómo realizarlo. El conjunto de estos sistemas de clasificación tiene en común que son jerárquicos y que sus análisis consideran las variables hidrológicas y geomorfológicas entre las más relevantes para establecer las categorías o tipologías (Malvares \& Lingua, 2004). A nivel global existen diversos sistemas de clasificación de humedales entre los que se pueden mencionar: Cowardin et al. 1979; Brinson 1993; Dugan 1993; Scott \& Jones 1995; Semeniuk \& Semeniuk 1995; Farinha et al. 1996; Finlayson et al., 2002; Keddy, 2010; Mitsch \& Gosselink 2007; entre otros. Las clasificaciones propuestas por Brinson (1993); Semeniuk \& Semeniuk (1995) y Finlayson et al. (2002) consideran las variables geomorfológicas e hidrológicas como las más relevantes para establecer las categorías principales. Por otro lado, es de amplio uso la clasificación de RAMSAR propuesta por Scott y Jones (1995), en la cual los factores que se tienen en cuenta son vegetación, geoforma, suelo e inundación (Brinson, 2004b; Malvárez \& Lingua, 2004). A su vez el Inventario de humedales del Mediterráneo (MedWet) de Farinha et al. (1996) es similar al sistema de RAMSAR.

La Secretaría de la Convención de Ramsar recomienda la elaboración de inventarios de humedales a los países que la integran, como instrumento de ayuda para la formulación de políticas nacionales de humedales (Molero \& Novelli, 2004). Argentina forma parte de esta Convención desde el año 1991 y en el año 1998 se realizó una propuesta de clasificación e inventariado elaborado por Canevari et al. (1998). En el año 2017 se efectuó una actualización del Inventario Nacional de Humedales (Nivel 1 a Escala 1:1.000.000 a 1:500.000), y se propone una caracterización ambiental de las distintas regiones y subregiones de humedales en Argentina, con un enfoque hidrogeomorfológico, que se relaciona estrechamente con el análisis integral de estos ecosistemas (Benzaquen et al., 2017).

Además de esta clasificaciones se realizaron otras para distintas regiones del país, en las que se han tenido en cuenta principalmente variables como suelos, pendientes, tipo de vegetación y geomorfología, tamaño, fluctuaciones hídricas, entre otras (Iriondo et al., 1974; Movia, 1984; Mazzonni \& Vázquez, 2004; Ahumada \& Faundez, 2009; Izquierdo et al., 2015; Izquierdo et al., 2016). Si bien se cuenta con estos estudios de base aún los estudios de clasificación y delimitación de humedales a escalas de mayor detalle son muy acotados (Benzaquen et al., 2017).

En Argentina, un tipo de humedal de montaña, situado a lo largo de la Cordillera de los Andes Centrales se denomina regionalmente vegas. Estos ambientes en general ocupan una proporción reducida del paisaje en relación al entorno desértico que los rodea. Sin embargo, tienen un relevante valor ecológico y socioeconómico en la región, por su elevada biodiversidad, productividad y función en el sistema hidrológico local (Amaya et al., 2019, Tapia Baldis et al., 2019, Izquierdo et al., 2015, 2016). Son un tipo de pradera siempreverde, sobre suelos hidromorfos, con áreas temporaria o permanentemente saturadas de agua, con vegetación 


\section{Ontivero et al. - Clasificación de humedales de montaña en los Andes Centrales}

herbácea e hidrofítica integrada por gramíneas y ciperáceas que forman molisoles, que se desarrollan generalmente en el piedemonte, valles, depresiones como lagunas y salinas (Martínez Carretero, 2007; Martínez Carretero \& Ontivero, 2017).

En la actualidad las técnicas de teledetección para el estudio de humedales son consideradas como la mejor herramienta para la identificación, cartografía y monitoreo de estos ecosistemas (Cartagena, 2002; Boyle et al., 2004; Mazzoni \& Vázquez, 2004; Mitsch \& Gosselink, 2007; Keddy, 2010; Otto et al., 2011). En los Andes Centrales, autores como Ontivero et al. $(2010,2011)$ Ontivero \& Martínez Carretero (2013), Martínez Carretero \& Ontivero, (2017), Izquierdo et al. (2015, 2016, 2018), Navarro et al. (2020), Pecker \& Trombotto (2021) han empleado con éxito técnicas de teledetección y trabajos de campo para evaluarlos.

Los ecosistemas de vegas de los Andes Centrales en los últimos años se han visto amenazados, por el incremento en la actividad minera, el sobrepastoreo y el cambio climático; sumado a la poca información disponible y el difícil acceso, hacen dificultosa su evaluación (Martínez Carretero \& Ontivero, 2017; Sosa \& Guevara, 2017). Esta situación destaca la importancia de desarrollar propuestas e investigaciones con uso de tecnologías de información geográfica con base en estudios de campo, que pueden ser utilizadas como herramientas para planificación, conservación y manejo.

El objetivo general de este trabajo es clasificar y caracterizar vegas en base a la geomorfología e hidrología en un sector de los Andes Centrales de Argentina. Los objetivos particulares son: a) identificar las vegas en el área de estudio; b) describir la hidrología según los tipos de escurrimiento; c) definir unidades geomorfológicas; d) clasificar las vegas según los tipos de escurrimiento y las unidades geomorfológicas donde se encuentran; f) caracterizar cada tipología según la hidrología y la geomorfología, superficie, altura y pendiente.

\section{Materiales y Métodos}

\section{Área de estudio}

El área de estudio ocupa una superficie de 570.405 ha e involucra a dos reservas naturales contiguas en los Andes Centrales de Argentina, la Reserva Provincial San Guillermo (RSG) (28 27’ S-69 32'O) en el sector noroeste de la provincia de San Juan, declarada Reserva Provincial en el 1972 y Reserva de Biosfera en el año 1980; y la Reserva Provincial Laguna Brava (RLB), en el sector suroeste de la provincia de La Rioja (28⒉ $\left.28^{\prime} \mathrm{S}-6^{\circ} 10^{\prime} \mathrm{O}\right)$, declarada área protegida en 1980 y sitio Ramsar en 2003 (Fig. 1). Ambas reservas se encuentran dentro de una de las zonas ecológicamente más intactas de América del Sur (Sanderson et al., 2002).

En relación al análisis geomorfológico, el área de estudio se enmarca dentro de la Unidad Andina. Según la clasificación de Sayago (1982) está conformada por las Provincias geológicas de la Cordillera Frontal, Puna, Precordillera Occidental y Sierras Pampeanas, y por las Regiones geomorfológicas de la sierra el Peñón, Punilla, Cordillera del Cajón de la Brea, Cordón del Infiernillo y Laguna La Brava. Geomorfológicamente, en el sector norte del área estudiada se ha identificado un paisaje con predominio de geoformas volcánicas, caracterizado por la presencia de conos, campos de lava, salinas y lagunas. En el sector sur, el paisaje está controlado por estructuras, con cordones montañosos limitados por fallas y valles intermontanos. Sin embargo, en el paisaje predominan actualmente los procesos exógenos tales como el fluvial y aluvial y en los sectores más elevados los de origen glacial, periglacial y fluvioglacial.

Desde el punto de vista geológico, el área pertenece a la Cordillera Frontal. El clima es frío, la temperatura media anual es de $0{ }^{\circ} \mathrm{C}$, la temperatura media mínima de $-6{ }^{\circ} \mathrm{C}$, la temperatura media máxima de $7^{\circ} \mathrm{C}$ y la precipitación anual entre 200-100 mm (Salvioli, 2007). Hidrológicamente el área pertenece a la subcuenca hidrográfica del río Blanco, tributaria de la cuenca del río Bermejo-Vinchina. Los ríos y arroyos permanentes como el Salado, Peña, Macho Muerto, La Brea y Santa Rosa, aportan sus aguas al río Blanco (Salvioli, 2007). El régimen dominante es de tipo nival, con los caudales directamente asociados a las temperaturas imperantes en las cuencas activas (Damiani, 2007; Salvioli, 2007).

En las cuencas hidrográficas de los Andes Centrales las vegas son el ecosistema más importante, ya que intervienen en los ciclos hidrológicos regulando los flujos hídricos superficiales y subterráneos (Méndez, 1986; Vich, 1996; Martínez Carretero \& Ontivero, 2017). El suministro hídrico de las vegas proviene del derretimiento de la nieve, deshielos glaciares y agua subterránea (Méndez, 1986; Canevari et al., 


\section{Área de estudio}
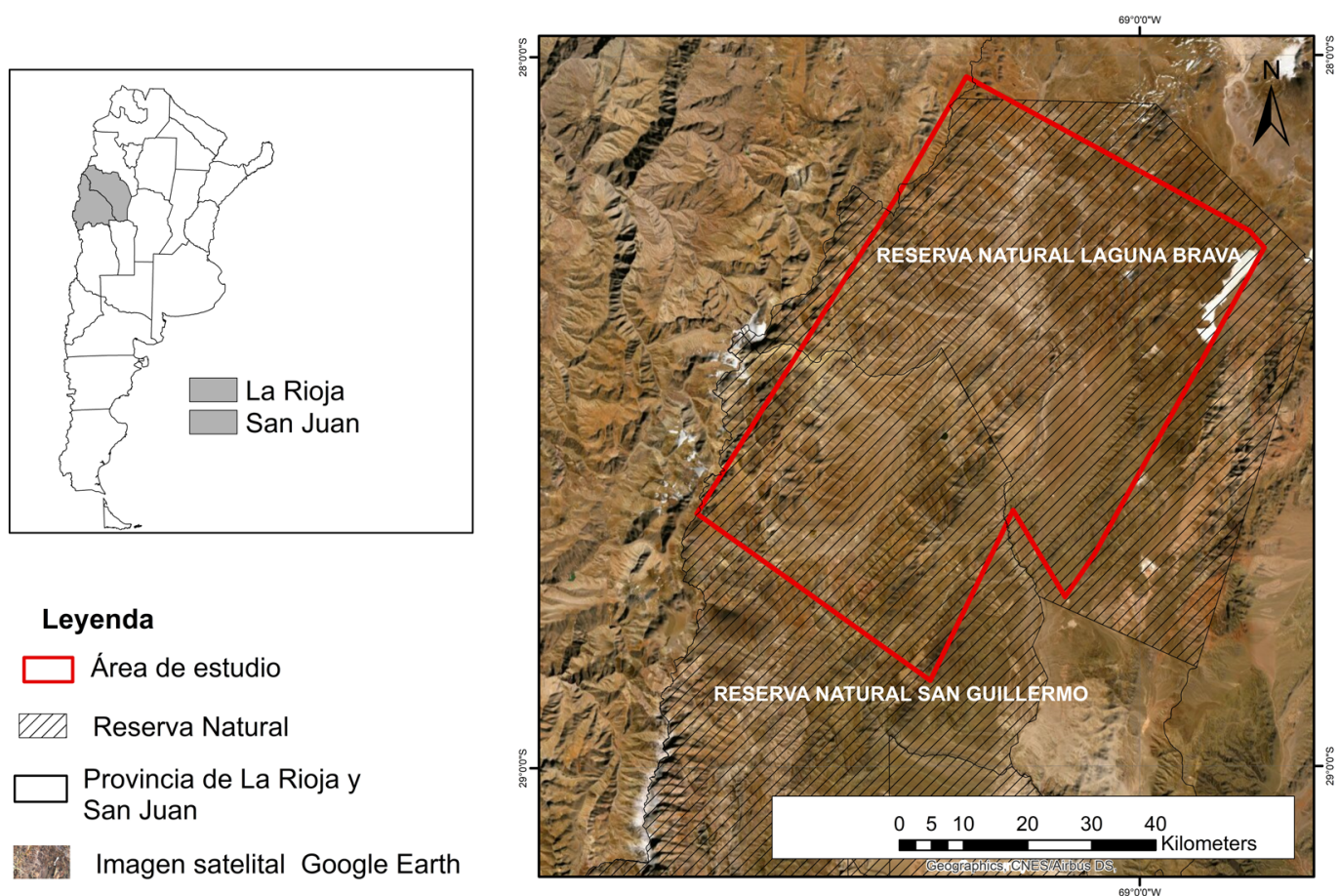

Fig. 1. Ubicación del área de estudio en un sector de los Andes Centrales, comprendido entre la Reserva Provincial San Guillermo, San Juan y Reserva Provincial Laguna Brava, La Rioja.

1998; Martínez Carretero, 2007; Martínez Carretero \& Ontivero, 2017). Las vegas en Argentina se distribuyen a lo largo de la región de la Puna y Altos Andes, entre los 3200 y $5200 \mathrm{msnm}$ aproximadamente (Izquierdo et al., 2015, 2016), y en el sector de los Andes Centrales se encuentran a menor altitud, entre los 2000 y 4300 msnm aproximadamente (Martínez Carretero \& Ontivero, 2017).

Las vegas son sitios de alta productividad y riqueza de especies vegetales, con suelos siempre húmedos que generan un ambiente reductor y por ende de acumulación de materia orgánica y carbono. Es notorio el contraste de este ecosistema, con el entorno montañoso que lo rodea (Martínez Carretero \& Ontivero, 2017).

\section{Metodología}

El método que se propone en este trabajo para determinar las distintas tipologías de vegas se basó en un enfoque biofísico integrado a distintas escalas de análisis del paisaje en el que se identificaron las vegas presentes en el área de estudio y se determinó su vinculación con aspectos hidrológicos y geomorfológicos. La identificación y digitalización de los distintos elementos se realizó mediante la técnica de análisis visual que no demanda altos requerimientos en equipamiento, software y capacitación técnica (Chuvieco, 2016). Además, esta técnica si bien requiere mayor trabajo de digitalización, permite tener mayor detalle en la identificación de las vegas. Este tipo de análisis visual es recomendable para la clasificación de vegas por parte de técnicos de organismos de gobierno en otras áreas no comprendidas en este estudio.

\section{Análisis de vega y aspectos hidrológicos}

Mediante la técnica de análisis visual de imágenes satelitales se llevó a cabo la identificación y digitalización de las vegas y de los distintos tipos de escurrimiento dominantes. Para ello se 


\section{Ontivero et al. - Clasificación de humedales de montaña en los Andes Centrales}

utilizaron 2 imágenes ALOS- AVNIR-2 de 10x10 m de resolución espacial, del 22/02/ 2008 con centro de longitud $-28,83-69,25$ y del 30/12/2008 con centro de longitud -28,24-68,86, con una combinación bandas RGB 4, 3, 2. Para el análisis visual de cada imagen se siguieron los criterios de Chuvieco (2006) tales como: el brillo, color, forma, tamaño, textura, sombras, contexto espacial, asociación y fenología a una escala de análisis 1:100.000.

Para identificar la vega se digitalizó en pantalla y determinó su localización y superficie. Se generó una cobertura (shape) con la digitalización de todas las vegas. Se determinó en hectáreas el tamaño máximo, mínimo y promedio de las vegas. Para el análisis de los aspectos hidrológicos se identificaron y se digitalizaron en pantalla los escurrimientos dominantes, siguiendo la clasificación de Vich (1996) donde se reconocen tres tipos: Superficial lineal; Subsuperficial mantiforme; Superficial lineal a subsuperficial mantiforme.

Se tuvo como información de base las coberturas de: cuenca hidrográfica; red de drenaje; ríos permanentes; ríos temporales; lagunas; glaciares cubiertos y descubiertos, provenientes del Sistema de Información Geográfico (SIG) del Atlas Socioeconómico de la Provincia de San Juan (Lizana et al., 2010) y de datos provistos por la Wildlife Conservation Society para la provincia de La Rioja a través de archivos digitalizados en un Disco Compacto en 2005 adquiridos por medio del Dr. Wrustten en el marco del proyecto Estepa Patagonica y Andina, Sector Reserva Natural Laguna Brava, provincia de La Rioja, Argentina.

Para determinar el tipo de escurrimiento dominante en cada una de las vegas se vincularon las coberturas: vegas y tipo de escurrimiento dominante, mediante un análisis espacial de superposición de coberturas (Bosque Sendra, 1992; Buzai \& Baxendale, 2011). De este análisis se obtuvo una cobertura nueva (vegastipo de escurrimiento). Se calculó el porcentaje de vegas vinculado a cada tipo de escurrimiento.

\section{Análisis de vega y aspectos geomorfológicos}

Para realizar la caracterización geomorfológica se utilizaron tres imágenes Landsat $5 \mathrm{TM}$ de $30 \times 30 \mathrm{~m}$ de resolución espacial del 12/09/2007 (path 232/ row 080 ) y $12 / 04 / 2007$ (path $233 /$ row 079 ; path 233 / row 080), donde se empleó una combinación de bandas RGB 7, 4, 2. La metodología siguió la técnica de interpretación visual a partir de la imagen satelital, con una escala de análisis de 1:250.000 teniendo en cuenta los criterios de Chuvieco (2006). Para identificar y delimitar las principales unidades geomorfológicas se siguieron las definiciones establecidas por Gutiérrez Elorza (2008); técnica ampliamente empleada en la región andina (Gonzalez Díaz y Fauqué, 1993; Suvires, 2000; Rosa y Mamaní, 2000; González et al., 2002; Perucca \& Esper Angillieri, 2008; Perucca, 2016). Además, se utilizó como información de base el mapa geomorfológico de San Juan (Suvires, 2000) y el mapa geomorfológico de La Rioja (Cisneros, 2000). Se generó una cobertura (shape) mediante la digitalización en pantalla de las unidades geomorfológicas identificadas.

Para determinar la unidad geomorfológica que contenía a cada una de las vegas se vincularon las coberturas de geomorfología y cobertura de vegas. La vinculación se realizó mediante un análisis espacial de superposición de coberturas (Bosque Sendra, 1992; Buzai \& Baxendale, 2011). Para determinar la asociación de las distintas unidades geomorfológicas con las vegas, se realizó una tabla de contingencia y un análisis de Chi cuadrado (Bosque Sendra, 1992). Se calculo el porcentaje de vegas registradas en las distintas unidades geomorfológicas.

Las corroboraciones en terreno de las unidades geomorfológicas, se realizaron durante las campañas de campo del verano del 2008 y 2009, mediante inspecciones visuales y fotográficas, siguiendo los caminos en el área de estudio. Además, se realizó inspección visual en campo en las 6 vegas seleccionadas para realizar los trabajos, determinando si se corresponde a la realidad de terreno con lo observado en las imágenes.

\section{Clasificación de vegas y caracterización de las} distintas tipologías

El patrón tipológico de cada vega se determinó mediante un análisis espacial de superposición de coberturas (Bosque Sendra, 1992; Buzai \& Baxendale, 2011), teniendo como base la cobertura de vega con tipo de escurrimiento dominante y con unidades geomorfológicas con vega. Para determinar la asociación, se confeccionó una tabla de contingencia y un análisis de Chi cuadrado (Bosque Sendra, 1992). Se determinaron los distintos patrones tipológicos de vegas y se generó un mapa de tipologías de vegas para toda el área de estudio.

Se caracterizó las distintas tipologías en base al escurrimiento dominante y unidades geomorfológicas. Se determinó la superficie y el número de vegas que se 
corresponde con cada tipología en el área de estudio. Los valores fueron expresados en porcentaje (\%). Además, se determinó si existen diferencias entre el tamaño, pendiente y altura entre las tipologías. Para determinar la pendiente y altura (msnm), se utilizó un Modelo Digital de Elevación Global de $15 \mathrm{~m}$ de resolución espacial proveniente de ASTER GDEM (https://asterweb.jpl.nasa.gov/gdem.asp). Para detectar las diferencias se calcularon los valores medios y se realizó un análisis de la varianza (ANAVA) de un factor. Como factor se consideró la variable tipo (cuatro tipos: de Ribera, Depresión, Ladera y Falla). Para comprobar la normalidad de los datos se aplicó la distribución con la prueba Kolmogorov-Smimov (Massey, 1951). Los datos de altura y superficie se transformaron mediante el método de raíz cuadrada. Se emplearon pruebas de comparaciones múltiples a posteriori, utilizando la prueba de Tukey.

Para el procesamiento de las imágenes satelitales, SIG y cartografía se empleó el programa de QGIS 2.18 Las Palmas (https://www.qgis.org/es/site/). Las imágenes satelitales se obtuvieron de la Comisión Nacional de Actividades Espaciales CONAE, https// www.argentina.gob.ar/ciencia/conae) e Instituto Nacional de Pesquisas Espaciales de Brasil (INPE, http://www.inpe.br/).

Todas las categorías evaluadas anteriormente fueron corroboradas en terreno mediante la inspección visual en 6 vegas de fácil acceso, durante las campañas del verano del 2008 y 2009. Las seis vegas seleccionadas estuvieron localizadas en distintas unidades geomorfológicas y con diferente tipo de escurrimiento dominante, tres en la Reserva Natural Laguna Brava: vega Quebrada Santo Domingo (28²6'29.36”S, 6850'54.32”W), vega Refugio Peñon (28 28'37.49'’S, 6850'11.63'W) y vega Laguna Brava (28 16 '29.76”S, 6849'28.45”W) y tres en la Reserva Natural San Guillermo: vega La

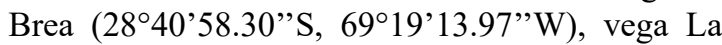
Guanaca $\left(28^{\circ} 43^{\prime} 52.89^{\prime \prime} \mathrm{S}, 6^{\circ} 17^{\prime} 4.02^{\prime \prime} \mathrm{W}\right)$ y vega Piuquenes $\left(28^{\circ} 44^{\prime} 10.92^{\prime \prime}\right.$ S, 69 $\left.9^{\circ} 7^{\prime} 4.08^{\prime \prime W}\right)$. Las vegas que no fueron corroboradas en campo se verificaron mediante análisis visuales con imágenes satelitales de alta resolución en Google Earth.

\section{Resultados}

Vega y aspectos hidrológicos

En el área de estudio se identificaron 304 vegas, con una superficie total cubierta por vegas de 2544 ha $(0,44 \%$ del área estudiada). La superficie mínima de la vega detectable por la metodología utilizada y la escala de trabajo fue de 0,1 ha, la superficie máxima de 140 ha y la superficie media de vega fue de 8 ha. El análisis de los distintos tipos de escurrimientos indica que el $50 \%$ de las vegas tiene un escurrimiento superficial lineal, el $40 \%$ de las vegas un escurrimiento superficial lineal a subsuperficial mantiforme y el $10 \%$ restante de las vegas presentan escurrimiento subsuperficial mantiforme,

\section{Vega y aspectos geomorfológicos}

Se identificó un total de 14 unidades geomorfológicas: Colada; Cono volcánico; Falla; Abanico aluvial; Ladera; Piedemonteindiferenciado; Planicie fluvial; Terraza fluvial, Depresión sin descarga superficial con o sin agua; Terraza fluvioglacial; Planicie fluvioglacial; Abanico fluvioglacial; Glaciar; Glaciar de escombro.

Se encontró una clara asociación entre las unidades geomorfológica y las vegas $\left(\mathrm{X}^{2}=798,34 ; \mathrm{df}=13 ; \mathrm{p}\right.$ $<0,05)$. Las unidades geomorfológicas que incluyen vegas son: Ladera, Piedemonte indiferenciado, Abanico aluvial, Planicie fluvial, Depresión sin descarga superficial con o sin agua y Falla. E1 50\% de las vegas está asociado a la Planicie fluvial, el 20\% a Abanico aluvial, el 10\% a Piedemonte indiferenciado y el 3\% a Ladera. La unidad geomorfológica Depresión sin descarga con o sin agua, se asocia al $12 \%$ de las vegas y sólo el $5 \%$ de vegas se encuentra sobre la unidad geomorfológica de Falla.

\section{Clasificación de Vegas}

Los patrones tipológicos se detectaron mediante la vinculación de las coberturas vega-tipo de escurrimiento dominante y unidad geomorfológicavega, se determinó que existe asociación entre el tipo de escurrimiento-vega y las unidades geomorfológicas-vega $\left(\mathrm{X}^{2}=2478,57\right.$; $\mathrm{df}=13$; $\mathrm{p}<$ $0,05)$. En la Tabla 1 se observa de acuerdo al análisis de cada asociación, a qué tipo de escurrimiento dominante con vega está vinculada cada unidad geomorfológica con vega.

Sobre la base de los resultados obtenidos se elaboró el patrón tipológico de vegas para los Andes Centrales, determinando cuatro tipologías: Vegas de ribera; Vega de ladera; Vega de depresión y Vega de Falla. Con esta información se elaboró un mapa de distribución de las distintas tipologías de vega 


\section{Ontivero et al. - Clasificación de humedales de montaña en los Andes Centrales}

Tabla 1. Tipo de escurrimiento dominante

asociado a la unidad geomorfológica en las 304 vegas del área de estudio.

\begin{tabular}{|ll|}
\hline \multicolumn{1}{|c|}{$\begin{array}{c}\text { Escurrimiento } \\
\text { Dominante }\end{array}$} & \multicolumn{1}{c|}{ Unidad Geomorfológica } \\
\hline $\begin{array}{l}\text { Superficial lineal } \\
\text { Superficial lineal } \\
\text { a subsuperficial } \\
\text { mantiforme }\end{array}$ & Planicie fluvial \\
$\begin{array}{l}\text { Subsuperficial } \\
\text { mantiforme }\end{array}$ & $\begin{array}{l}\text { Abanico aluvial, } \\
\text { Ladera, Piedemonte } \\
\text { indiferenciado y Falla }\end{array}$ \\
\hline
\end{tabular}

determinadas en el área de estudio (Fig. 2).

\section{Caracterización de las distintas tipologías}

A partir de las características hidrológicas y geomorfológicas, las cuatro tipologías de vegas identificadas quedan definidas de la siguiente manera:

- Vegas de rivera: Están localizadas sobre planicies fluviales, que incluyen el curso del río y la llanura de inundación adyacente, presentan escurrimiento superficial lineal dominante. El escurrimiento puede ser permanente o temporal. Reciben principalmente aportes superficiales provenientes del derretimiento de mantos de nieves, glaciares y precipitaciones níveas (Fig. 3A).

- Vegas de ladera: Se localizan sobre laderas montañosas, abanicos aluviales y piedemontes indiferenciados, presentan escurrimiento superficial lineal a subsuperficial mantiforme dominante en la zona deprimida. Pueden tener agua permanente o temporal. Reciben principalmente aportes de agua subterránea y en ocasiones aportes superficiales temporales (Fig. 3B).

-Vegas de depresión: Se localizan sobre depresiones sin descarga superficial con o sin agua, donde domina el escurrimiento subsuperficial mantiforme. Se consideran dentro de esta categoría las vegas, lagunas y salinas con agua permanente o temporal. Su alimentación proviene principalmente del agua subterránea; aunque en ocasiones puede recibir aportes superficiales (Fig. 3C).

-Vegas de falla: Se ubican alineadas a lo largo de fallas geológicas, presentan escurrimiento superficial lineal a subsuperficial mantiforme dominante en la zona deprimida o labio bajo de la falla pueden tener o no agua en superficie y ésta ser permanente o temporal. Reciben principalmente aportes de agua subterránea y en menor medida aportes superficiales (Fig. 3D).

Las Vegas de ribera fueron las más frecuentes en la zona de estudio, representando el $50 \%$ de las vegas mapeadas, seguidas de las Vegas de ladera (33\%), Vegas de depresión (12\%) y Vegas de falla (5\%). Asimismo, las Vegas de ribera representaron un $74 \%$ de la superficie mapeada de vegas, seguidas por las Vegas de ladera (12\%), Vegas de falla (9\%) y Vegas de depresión (5\%). (Fig. 4).

En relación al tamaño se detectaron diferencias significativas entre las distintas tipologías de vega $(\mathrm{F}=4,997 ; \mathrm{P}=0,002 ; \mathrm{N}=304)$. Las Vegas de ribera y Vegas de falla son las de mayor tamaño y se

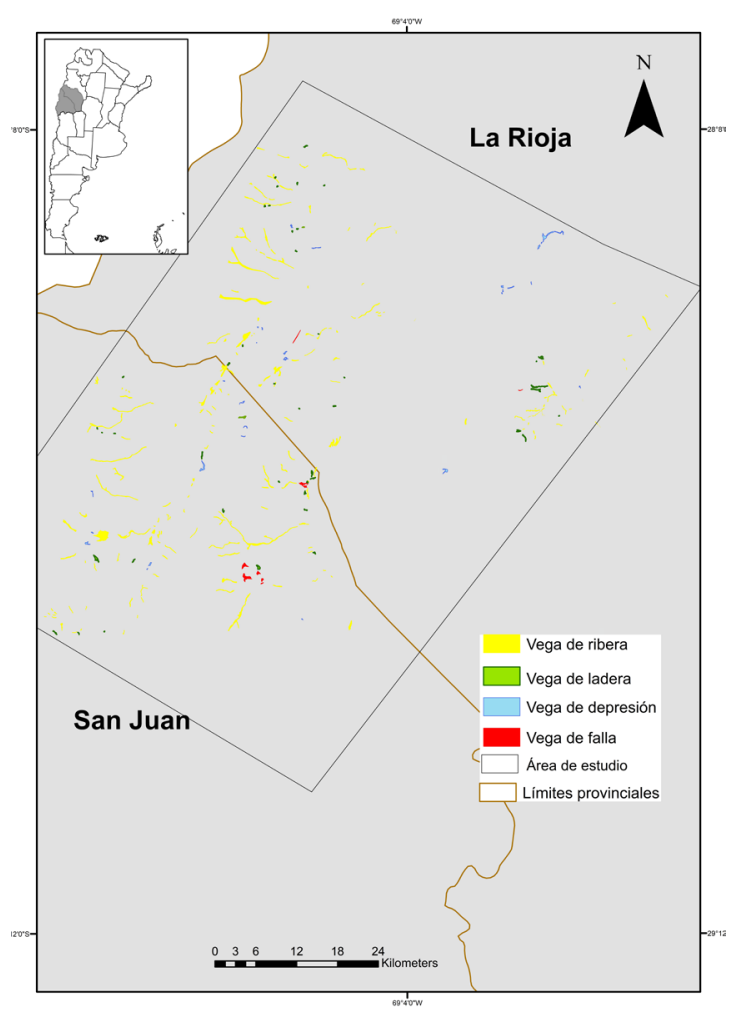

Fig. 2. Mapa de distribución de las distintas tipologías de vegas: Vega de ribera, Vega de ladera, Vega de depresión, Vega de falla en un sector de Ios Andes Centrales, comprendido entre la Reserva Provincial San Guillermo, San Juan y Reserva Provincial Laguna Brava, La Rioja. 
Bol. Soc. Argent. Bot. 57 (1) 2022

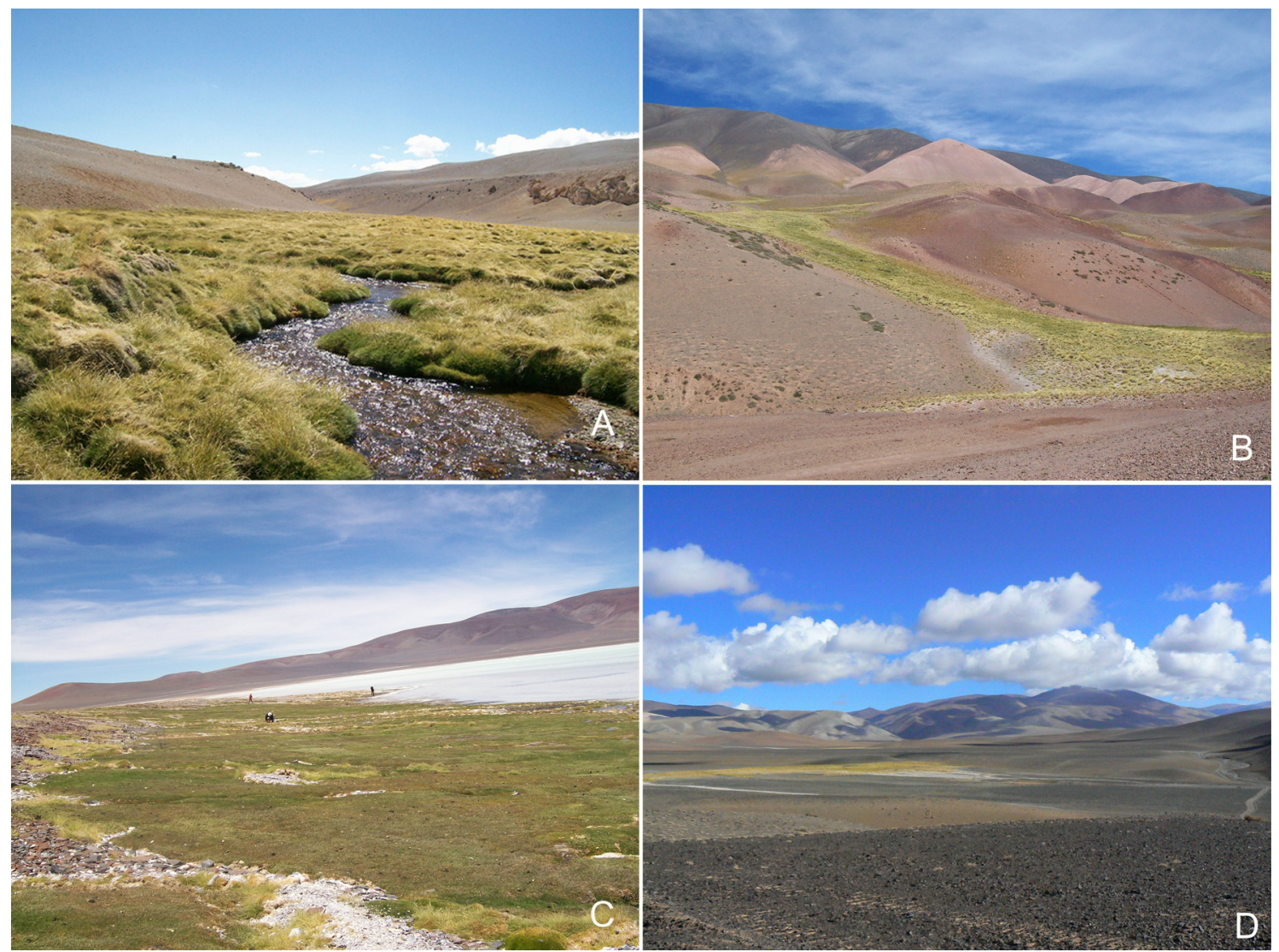

Fig. 3. Cuatro tipologías de vega caracterizadas en un sector de los Andes Centrales, comprendido entre la Reserva Provincial San Guillermo, San Juan y Reserva Provincial Laguna Brava, La Rioja. A: Vega de ribera, B: Vega de ladera, C: Vega de depresión, D: Vega de falla.

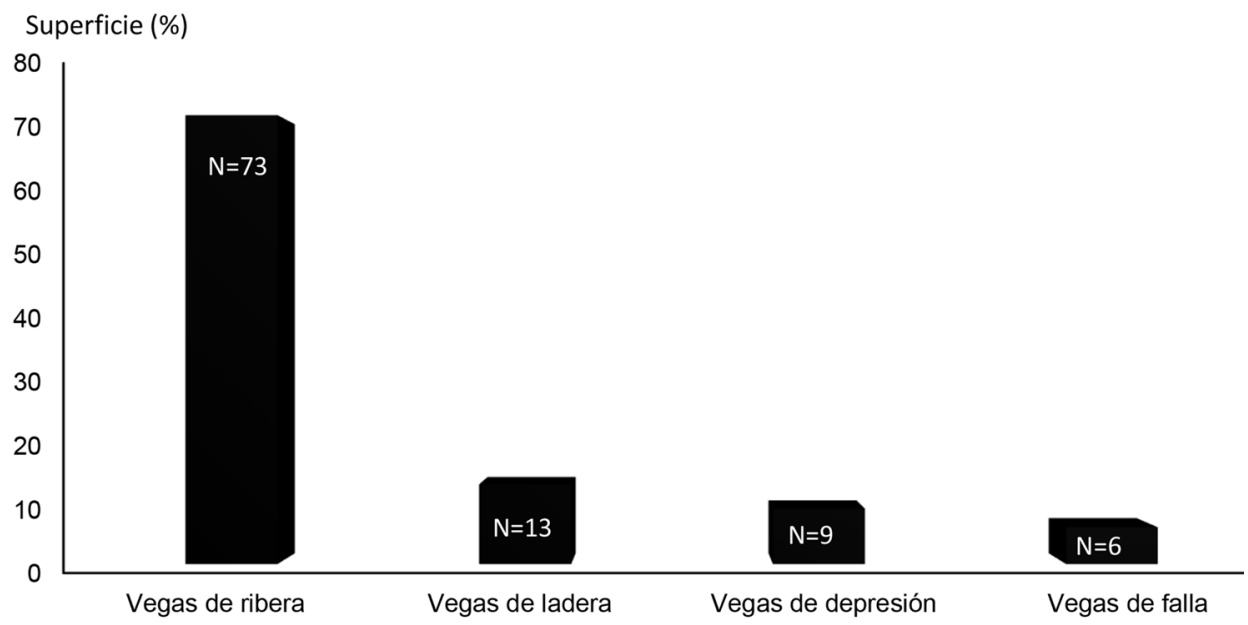

Fig. 4 Superficie relativa y frecuencia de vegas en cada una de las cuatro tipologías de vegas observadas en un sector de los Andes Centrales, comprendido entre la Reserva Provincial San Guillermo, San Juan y Reserva Provincial Laguna Brava, La Rioja. 


\section{Ontivero et al. - Clasificación de humedales de montaña en los Andes Centrales}

diferencian de las Vegas de ladera que son las de menor tamaño (test a posteriori Tukey $\mathrm{P}=<0,05$ ). Las Vegas de depresión, en relación a las restantes tipologías tienen un tamaño intermedio. Si bien se observaron también diferencias en la altura y la pendiente, éstas no resultaron significativas entre las distintas tipologías.

\section{Discusión y Conclusión}

La geomorfología y la hidrología son factores relevantes para clasificar humedales, por lo que han sido utilizados en varios sistemas de clasificación internacionales y nacionales como Cowardin et al. (1979), Secretaría de Convención de Ramsar (1990), Brinson (1993), Movía (1984), Iriondo et al. (1974) y Benzaquen et al. (2017). En este trabajo, la propuesta de clasificación de Vegas en los Andes Centrales de Argentina se realizó a base de un enfoque biofísico integrado a distintas escalas de análisis, teniendo en cuenta la localización de la vega, los factores geomorfológicos e hidrológicos. A escala de paisaje se definieron distintos tipos de vegas determinados por la unidad geomorfológica (Planicies fluviales, Abanico aluvial, Ladera, Piedemonte indiferenciado, Depresión sin descarga superficial con o sin agua y Falla) y el tipo de escurrimiento dominante (Superficial lineal, Subsuperficial mantiforme y Subsuperficial mantiforme a superficial lineal). Considerando la totalidad de Vegas incluidas en el análisis se reconocen cuatro tipologías: de ribera, ladera, depresión y de falla. Las Vegas de ribera y de ladera fueron las predominantes en cuanto a frecuencia, cantidad de superficie y tamaño. En menor medida se ubican las Vegas de depresión y de falla; si bien las Vegas de falla tiene gran tamaño fueron menos frecuente en el área.

Cada tipología está definida por una unidad geomorfológica en particular y un tipo de escurrimiento dominante, como es el caso de las Vegas de ribera, de depresión y de falla. Las Vegas de ladera se encuentran asociadas a varias unidades geomorfológicas, tales como Abanico aluvial, Ladera y Piedemonte indiferenciado, todas estas unidades comparten el mismo tipo de escurrimiento dominante Superficial lineal a subsuperficial mantiforme. Este grupo de unidades geomorfológicas son similares por estar dentro de la unidad morfoestructural de montaña (Gutiérrez Elorza, 2008) y han sido agrupadas en una misma categoría. La tipología de Vega de falla no ha sido incluida en ninguna de las clasificaciones de humedales vigentes hasta el momento, cabe diferenciarla y destacarla dado que las fallas son habituales en la zona de los Andes Centrales debido a que en este sector se concentran las principales evidencias de deformación tectónica cuaternaria (fallas y pliegues), producto de la convergencia entre las placas de Nazca y América del Sur (Anderson et al., 2007; Perucca \& Vargas, 2014).

En cuanto a la tipología de Vega de ribera propuesta en este trabajo, la misma considera tanto a la Planicie fluvial como al cauce del río, ya que las vegas se pueden encontrar en ambos sectores, debido a las variaciones topográficas y a su vegetación herbácea e hidrofítica característica (Martínez Carretero \& Ontivero, 2017); similar a la propuesta de Brinson (1993) en su clasificación de humedales de Ribera incluye la planicie y el cauce del río. Sin embargo, difiere del grupo fluvial de Cowardin et al. (1979) y de la Convención de Ramsar (2005), que es definida sólo para los cauces de ríos.

Otro aspecto que se ha tenido en cuenta en este trabajo es que el tipo de escurrimiento dominante puede ser permanente o temporal, ya que las condiciones hídricas en estos ambientes son muy fluctuantes (Izquierdo et al., 2016). Las Vegas presentan cambios ante las fluctuaciones hídricas, que pueden estar vinculadas a causas antrópicas, como la minería y el pastoreo (Reboratti, 2006; Izquierdo et al., 2016;) y climáticas (Caziani y Derlindati, 1999; Izquierdo et al., 2016). La frecuencia y superficie de cada tipología varía entre vegas, siendo las Vegas de ribera las más frecuentes y con mayor superficie en el área de estudio. Este predominio en número y superficie de las Vegas de ribera fue también observado en humedales (mallines) en Santa Cruz (Mazzonni \& Vázquez, 2004).

La aplicación de esta propuesta metodológica a nivel regional contribuirá a la gestión ambiental, conservación y monitoreo de estos ecosistemas de alto valor ecológico y socioeconómico en la región. Si bien la clasificación propuesta es de fácil implementación, al ser manual requiere de esfuerzos para ser aplicado en grandes extensiones. La clasificación de las distintas tipologías servirá de base para plantear estudios más detallados en el 
comportamiento de los patrones tipológicos ante posibles escenarios de cambios, donde el suministro de agua puede variar debido a condiciones climáticas o al aumento de las actividades antrópicas. Esto se debe a que los humedales de zonas desérticas pueden ser afectados seriamente si se extrae más agua de la que se renueva, ya sea directamente del humedal, por extracción de agua subterránea, o no dejando que el agua llegue al mismo, por desvío de los escurrimientos.

\section{Contribución de los Autores}

MO y EM diseño y realización de la investigación, análisis, recolección, interpretación de datos, preparación de las figuras y redacción del manuscrito. LP diseño y realización de la investigación, análisis, interpretación de datos y revisión del manuscrito.

\section{Agradecimientos}

A Yanina Ripoll, Dardo Recabarren y guardaparques del Dirección de Conservación y Áreas Protegidas de la Secretaría de Ambiente y Desarrollo Sustentable de la provincia de San Juan; a la Comisión Nacional de Actividades Espaciales, Argentina especialmente a Graciela Salinas de Salmuni. Al CEFOCCA-UNSJ C. Lizana; W. Melian; M. del Cid y G. Garces y al Grupo de Geobotánica y Fitogeografía del IADIZA-CONICET. Agradecemos los aportes realizados por el editor y revisores anónimos que evaluaron el trabajo, en particular a la Dra. Izquierdo.

Estudio apoyado por la 021/18 Resol. Universidad Nacional de San Juan bajo la dirección de E:MC.

\section{Bibliografía}

AHUMADA, M. \& L. FAÚNDEZ. 2009. Guía Descriptiva de los sistemas vegetacionales azonales hídricos terrestres de la ecorregión altiplánica (SVAHT). Ministerio de agricultura de Chile, Servicio Agrícola y Ganadero. Santiago. Chile.

AMAYA, N., D. BLANCO, A. CHAMORRO, J. GONNET, C. HEGOBURU \& H. SOSA. 2019. Conservación y manejo de vegas altoandinas en
Argentina y Perú. Dos estudios de caso. Fundación para la conservación y el uso sustentable de los Humedales. Wetlands International.

ANDERSON, M., P. ALVARADO, G. ZANDT \& S. BECK. 2007. Geometry and brittle deformation on the subducting Nazca Plate, central Chile and Argentina. Geophysical Journal International 171: 419-434. https://doi.org/10.1111/j.1365-246X.2007.03483.x

BENZAQUEN, L., D.E. BLANCO, R. BO, P. KANDUS, G. LINGUA, P. MINOTTI \& R. QUINTANA. 2017. Regiones de humedales de la Argentina. Ministerio de Ambiente y Desarrollo Sustentable, Fundación Humedales/Wetlands International. Universidad Nacional de San Martín y Universidad de Buenos Aires.

BOSQUE SENDRA, J. 1992. Sistemas de Información Geográfica. Ediciones Rialp S.A, Madrid.

BOYLE, T. P., S. M. CAZIANI \& R. G. WALTERMIRE. 2004. Landsat TM inventory and assessment of waterbird habitat in the southern altiplano of South America. Wetlands Ecology and Management 12: 563-573. https://doi.org/10.1007/s11273-005-1761-2

BRINSON, M.M. 1993. A hydrogeomorphic Classification for Wetlands. Technical Report WRPDE-4. Waterways Experiment Station, Army Corps of Engineers, Vicksburg, MS. Washington, DC.

BRINSON, M. 2004. a. Niveles extremos de variación de patrones y procesos en humedales. En: MALVÁREZ, A. I (ed.), Documentos del cursotaller: Bases ecológicas para la clasificación e inventario de humedales en Argentina, pp. 19-4. $1^{\circ}$ Edición, Buenos Aires.

BRINSON, M. 2004. b. Conceptos y desafíos de la clasificación de humedales. En: MALVÁREZ, A. I (Ed.), Documentos del curso-taller: Bases ecológicas para la clasificación e inventario de humedales en Argentina, pp. 25-36. $1^{\circ}$ Edición, Buenos Aires.

BRINSON, M. 2011. Clasificación de los Humedales. En: B. A. LEPAGE (ed.), Humedales. Editorial Springer Países Bajos.

BUZAI, C. \& C. BAXENDALE. 2011. Análisis socioespacial con sistema de información geográfica : Perspectivas cientificas: temáticas de base raster. Lugar Editorial S. A., Buenos Aires. Argentina.

CANEVARI, P., D.E. BLANCO, E.H. BUCHER, G. CASTRO \& I. DAVIDSON. 1998. Los humedales de la Argentina. Clasificación, situación actual, conservación y legislación. Wetlands International, Buenos Aires. Argentina. 


\section{Ontivero et al. - Clasificación de humedales de montaña en los Andes Centrales}

CARTAGENA, F. D. 2002. Identificación y análisis recambios en bofedales de la cordillera occidental y altiplano de Bolivia. Tesis maestría profesional en Levantamiento de Recursos Hídricos. Manejo y conservación de cuencas. Centro latinoamericano aeroespacial y aplicaciones SIG para el desarrollo sostenible de los recursos naturales CLAS.

CANEVARI, P., D.E. BLANCO, E.H. BUCHER, G. CASTRO \& I. DAVIDSON. 1998. Los humedales de la Argentina. Clasificación, situación actual, conservación y legislación. Wetlands International, Buenos Aires. Argentina.

CHUVIECO, E. 2006. Teledetección ambiental. La observación de la tierra desde el espacio. $1^{\circ}$ Edición. Editorial Ariel, S.A. Barcelona. España.

COWARDIN, L.M., V. CARTER, F.C. GOLET \& E.T. LAROE 1979. Classification of wetlandsand deepwater habitats of the United States. U. S. Department of the Interior, Fishand Wildlife Service, Washington, D.C.

DAMIANI, O. 2007. Aguas superficiales. En: MARTÍNEZ CARRETERO, E. (ed.), Diversidad biológica y cultural de los altos Andes Centrales de Argentina: linea de base de la Reserva Biósfera San Guillermo, San Juan, pp.101-112. $1^{\circ}$ Edición. Editorial Universidad Nacional de San Juan, San Juan. Argentina.

DUGAN, P. 1993. Wetlands in danger. Oxford University Press, New York, New York, USA.

FARINHA, J.C., L.T. COSTA, A. MANTZAVELAS, E. FITOKA, N. HECKER \& P. TOMAS VIVES.1996. Mediterranean wetland inventory: Habitat Description System. MedWet Publication Instituto da Conservação da Natureza, Lisboa, Wetlands International, Slimbridge and EKBY, Thessaloniki.

FINLAYSON, C., N. DAVIDSON, A. SPIERS \& N. STEVENSON. 1999. Global wetland inventorycurrent status and future priorities. Mar. Freshwat. Res. 50: 717-727. https://doi.org/10.1071/MF99098

FINLAYSON, C., G. W. BEGG, J. HOWES, J. DAVIES, K. TAGI \& J. LOWRY. 2002. A manual for an inventory of Asian wetlands. Version 1.0. Wetlands International Global, Series 10, Kuala Lumpur, Malasia.

GUTIÉRREZ ELORZA, M. 2008. Geomorfología. $1^{\circ}$ Edición Pearson/Prentice Hall, Madrid.

IRIONDO, M., J. A. ORELLANA \& J. J. NEIFF. 1974. Sobre el concepto de mallín cordillerano. Revista Asoc. Cientif. Nat. Lit. 5: 45-52. https://doi.org/10.14409/natura.v1i5.3275
IZQUIERDO A., J. FOGUET \& H. RICARDO GRAU. 2015. Mapping and spatial characterization of Argentine High Andean peatbogs. Wetlands Ecology and Management 23: 963-976. https://doi.org/10.1007/s11273-015-9433-3.

IZQUIERDO A., J. FOGUET \& H. RICARDO GRAU. 2016. Hidroecosistemas de la Puna y Altos Andes de Argentina. Acta Geológica Lilloana. 28: 390-402.

KEDDY, P. A. 2010. Wetland Ecology. Principles and Conservation. $2^{\circ}$ Edition. Editorial Cambridge UniversityPress, New York. https://doi.org/10.1017/CBO9780511778179

LIZANA, C., M. MARTINEZ, E. MARQUEZ, M. DEL CID, N. HERRERA, G. GARCES OLSEN, I. MALLAMACI, J. MARAZ, V. OVIEDO, R. VELAZQUEZ \& M. MARTINELLI 2010. Atlas Socioeconómico Provincia de San Juan. Centro de Fotogrametría Cartografía y Catastro. Universidad Nacional de San Juan.

MALVÁREZ, A. 2004. Consideraciones preliminares sobre un sistema nacional de clasificación e inventariado de humedales. En: MALVÁREZ, A. I (ed.), Documentos del curso-taller: Bases ecológicas para la clasificación e inventario de humedales en Argentina, pp. 13-15. $1^{\circ}$ Edición, Buenos Aires. Argentina.

MARTÍNEZ CARRETERO, E. 2007. Diversidad biológica y cultural de los altos Andes Centrales de Argentina: línea de base de la Reserva Biósfera San Guillermo, San Juan. Edición literaria a cargo de Eduardo Martínez Carretero. $1^{\circ}$ Edición. Universidad Nacional de San Juan, San Juan. Argentina.

MARTÍNEZ CARRETERO, E. \& M. ONTIVERO 2017. Vegas. Ecosistema altoandino de importancia biológica, ecológica y socio-económica. En: MARTÍNEZ CARRETERO, E.\&A. GARCÍA(ed.), San Juan Ambiental. pp. 199-213. Universidad Nacional de San Juan, San Juan. Argentina.

MASSEY JR, F. J. 1951. The Kolmogorov-Smirnov test for goodness of fit. Journal of the American statistical Association 46: 68-78.

MAZZONI, E. \& M. VAZQUEZ. 2004. Ecosistemas de mallines y paisaje de la Patagonia Austral, provincia de Santa Cruz. Universidad Nacional de la Patagonia Austral. Unidad Académica de Río Gallego, Convenio EEA. Santa Cruz, INTA. Ediciones INTA, Buenos Aires. Argentina.

MÉNDEZ, E. 1986. Dinamismo de la vegetación en el Valle Superior del Río Atuel. Vegas y Conos de deyección. Parodiana 4: 333-350. 
NAVARRO, C., A. IZQUIERDO, E. ARÁOZ, J. FOGUET \& H. RICARDO. GRAU. 2020. Rewilding of large herbivore communities in high elevation Puna: geographic segregation and no evidence of positive effects on peatland productivity. Reg. Environ. Change 20:112. https://doi.org/10.1007/s10113-020-01704-8

MÉNDEZ, E. 2004. La Vegetación de los altos Andes I. Pisos de vegetación del flanco oriental del cordón del Plata (Mendoza, Argentina). Bol. Soc. Argent. Bot. 39: 227-253. https://doi.org/10.31055/1851.2372.v49.n2.7857

MITSCH, W. J. \& J.G. GOSSELINK. 2007. Wetlands. $4^{\circ}$ Edition. Editorial John Wiley y Sons, Inc, Hoboken, New Jersey.

MOLERO, G. C. \& Y. S. NOVELLI. 2004. Un sistema de clasificación de humedales propuesto para la Convención de Ramsar. En: MALVÁREZ, A. I (ed.), Documentos del curso-taller: Bases ecológicas para la clasificación e inventario de humedales en Argentina, pp. 37-51. $1^{\circ}$ Edición, Buenos Aires. Argentina.

MOVIA, C. 1984. Tipología de mallines. En: RABASSA, J. \& BRANDANI, A., CAPUA, O.Y OTTONELLO, E. Cursos de campo en sistemas ecogeomorfológicos: Guía de excursiones. Dpto de Posgrado. Universidad Nacional del Comahue. Neuquén.

ONTIVERO, M., E. MARTÍNEZ CARRETERO, C. LIZANA \& G. SALINAS SALMUNI 2011. Relevamientos de humedales altoandinos (vegas) en la provincia de San Juan, mediante Teledetección y SIG. Argentina. XXXIII Jornadas Argentinas de Botánica. Bol. Soc. Argent. Bot. 46 (Supl): 202-203.

ONTIVERO, M. \& E. MARTÍNEZ CARRETERO. 2013. El ecosistema de vega en el corredor bioceánico (San Juan, Argentina) mediante el empleo de TIG. En GARCÍA, A. (ed.), El Corredor Bioceánico en San Juan. Recursos culturales y naturales del sector andino, pp. 23-30. Editorial Universidad Nacional de San Juan, San Juan. Argentina.

OTTO, M., D. SCHERER \& J. RICHTERS. 2011. Hydrological differentiation and spatial distribution of high altitude wetlands in a semi-arid Andean region derived from satellite data. Hydrology and Earth System Sciences 15: 1713-1727. https://doi.org/10.5194/hess-15-1713-2011

PECKER MARCOSIG, I. \& D. TROMBOTTO LIAUDAT. 2021. Análisis de la dinámica de dos mallines de altura en Vallecitos, Cordón del Plata,
Mendoza, Argentina, en el periodo 2002-2019. Acta Geológica Lilloana 33: 1-24.

https://doi.org/10.30550/j.agl/2021.33.1/2021-02-18

PERUCCA, L. 2016. Geomorfología de San Juan. En: MARTÍNEZ CARRETERO, E. \& A. GARCÍA (ed.), San Juan Ambiental pp. 95-116. Universidad Nacional de San Juan, San Juan. Argentina.

PERUCCA, L. \& M.Y. ESPER ANGILLIERI. 2008. A preliminary inventory of periglacial landforms in the Andes of La Rioja and San Juan, Argentina, at about $20^{\circ}$ S. Quaternary International, 190: 171-179. https://doi.org/10.1016/j.quaint.2007.10.007

PERUCCA, L. \& N. VARGAS. 2014. Neotectónica de la Provincia de San Juan, centro-oeste de Argentina. Boletín de la Sociedad Geológica Mexicana 66: 291304. https://doi.org/10.18268/BSGM2014v66n2a6

QGIS, 2016. "Las Palmas" Development Team. QGIS Geographic Information System. Open Source Geospatial Foundation Project [online]. Disponible en: http://qgis.osgeo.org [Acceso: 10 julio 2021].

SALVIOLI, G. H. 2007. Caracterización hidrometeorológica. En: MARTÍNEZ CARRETERO, E. (ed.), Diversidad biológica $y$ cultural de los altos Andes Centrales de Argentina: línea de base de la Reserva Biósfera San Guillermo, San Juan, pp.61-87. $1^{\circ}$ Edición. Editorial Universidad Nacional de San Juan, San Juan. Argentina.

SANDERSON, E. W., M. A. JAITEH, K. H. LEVY, A. REDFORD, V. WANNEBO \& G. WOOLMER. 2002. The human footprint and the last of the wild. Bioscience 52: 891-904. https://doi.org/10.1641/00063568(2002)052[0891:THFATL]2.0.CO;2

SCOTT, D.A. \& T. A. JONES. 1995. Classification and inventory of wetlands: A global overview. Vegetatio 118: 16-33. https://doi.org/10.1007/BF00045186

SECRETARÍA DE LA CONVENCIÓN DE RAMSAR. 2008. Convención de Ramsar y Grupo de Contacto EHAA. 2008. Estrategia Regional para la Conservación y Uso Sostenible de Humedales Altoandinos. CONDESAN y TNC-Chile. Gobiernos de Ecuador y Chile.

SECRETARÍA DE LA CONVENCIÓN DE RAMSAR. 2005. Ramsar COP9 DOC. 26. Estrategia Regional de Conservación y Uso Sostenible de los Humedales Altoandinos. 9a Reunión de la Conferencia de las Partes Contratantes en la Convención sobre los Humedales (Ramsar, Irán, 1971). Kampala, Uganda.

SEMENIUK, C.A. \& V. SEMENIUK. 1995. A geomorphic approach to global wetland classification. Vegetatio 118 : 103-124. https://doi.org/10.1007/BF00045193 


\section{Ontivero et al. - Clasificación de humedales de montaña en los Andes Centrales}

SOSA H. \& B. GUEVARA. 2017. Subregión Vegas y lagunas altoandina. En: BENZAQUEN, L., D.E. BLANCO, R. BO, P. KANDUS, G. LINGUA, P. MINOTTI \& R. QUINTANA (ed.). Regiones de humedales de la Argentina, pp.83-92. Ministerio de Ambiente y Desarrollo Sustentable, Fundación Humedales/Wetlands International. Universidad Nacional de San Martín y Universidad de Buenos Aires.
TAPIA BALDIS, C., D. TROMBOTTO LIAUDAT \& A. AHUMADA. 2019. Ambiente periglacial y regiones geocriológicas en los Andes de San Juan (28 $\left.-33^{\circ} \mathrm{S}\right)$. Revista de la Asociación Geológica Argentina 76: 46-63.

VICH, A. 1996. Aguas Continentales Formas y Procesos. Mendoza, Impreso en los talleres gráficos del centro de economía legislación y administración del agua y el ambiente. Argentina. 
